\title{
Management and horse-level risk factors for recurrent colic in the UK general equine practice population
}

\author{
C.E. Scantlebury ${ }^{1}$, D.C. Archer ${ }^{1}$, C.J. Proudman ${ }^{2}$, \& G.L. Pinchbeck ${ }^{1}$ \\ ${ }^{1}$ Institute of Infection and Global Health, Department of Epidemiology and Population Health, \\ School of Veterinary Science, University of Liverpool, Leahurst campus, Neston, Wirral CH64 7TE \\ ${ }^{2}$ School of Veterinary Medicine, Faculty of Health and Medical Sciences, University of Surrey, Guildford, \\ Surrey. GU2 7TE \\ Corresponding author email: claire.scantlebury@liverpool.ac.uk
}

\begin{abstract}
Summary
Reasons for performing study: Recurrent colic occurs frequently in the general horse population but little evidence exists about what factors place horses at greater risk of recurrent colic.
\end{abstract}

Aims: To quantify time-varying and non time-varying risk factors for recurrent colic among horses attended by first-opinion veterinary surgeons in NW England.

Methods: A nested case - control study was conducted on data from a prospective longitudinal cohort study of 127 horses recruited subsequent to an episode of medical colic. Data were collected on management and recurrent colic episodes at 4 monthly intervals by telephone questionnaires. All recurrent colic episodes were selected as cases; controls were unmatched and randomly selected from all horse time at risk. Data relating to the 30 days prior to the date of colic or control selection were used to determine exposure status. Multivariable logistic regression analyses were used to determine risk factors for recurrent colic.

Results: In total, 59 cases and 177 controls were included. The final model showed that horses that displayed crib-biting/windsucking (CBWS) (OR 10.1, 95\% CI 2.5, 41.0) or weaving behaviour (OR 3.9, 95\% CI 1.5, 10.1) had an increased risk of recurrence of colic. Increasing time at pasture reduced the risk of recurrence (OR $0.99,95 \%$ CI $0.99,1.0)$. A significant interaction was found suggesting the risk 
associated with CBWS may be modified by feeding fruit/vegetables, however further research is required before recommendations for feeding practices can be made.

Conclusions and potential relevance: This study suggests that sufficient access to pasture may be an important means of reducing recurrent colic risk. The behavioural risk factors highlight individuals who may be at increased risk of recurrent colic and whose colic prevention strategies should be carefully managed.

\section{Introduction}

Colic is a high priority health concern for horse-owners [1]. It occurs frequently and may result in the need for surgical intervention or the death of the horse $[2,3]$. Whilst many colic cases are isolated episodes, recurrence is frequently reported (following both medical and surgical colic episodes) and has been identified as a frustrating problem to manage $[4,5]$. In a previous study, the rate of recurrent colic in the general horse population was shown to be high at 50 episodes of recurrence per hundred horse-years-at-risk [6].

42 Previous epidemiological studies have identified factors associated with altered

43 likelihood of colic. These have included season and a number of management and 44 horse-level risk factors including; age and breed, crib-biting/windsucking (CBWS)

45 behaviour, parasites, dietary type and feeding practices, exercise, stabling and access

46 to pasture, access to water, transportation, vaccination, premises/owner factors and 47 use of the horse [7]. These findings highlight the multifactorial nature of colic and 48 horses that have suffered an initial colic episode have been shown to be at increased risk of further colic episodes [8, 9, 10, and 11].

At present, there is little evidence available of horse and management level risk factors associated with increased risk of recurrent colic. One study of horses seen by ambulatory veterinarians in Texas compared horses with recurrent colic with two control populations; horses with colic but no prior history of colic and horses without colic (or prior history of colic) [12]. Risk factors associated with recurrent colic were; previous abdominal surgery, age ( $>8$ years), feeding coastal grass hay, recent change in diet, low stocking density $(<0.5$ horses/acre), geldings and the Arabian

57 breed. This study gave important indications of risk factors for recurrent colic, although due to the retrospective case-control study format, it was not able to 
59 investigate temporal (time-varying) relationships between the exposures (e.g.

management practices at that time) and risk of recurrent colic.

61 There is growing evidence to implicate a link between equine stereotypic behaviour

62 and increased colic risk. Studies investigating both epiploic foramen entrapment

63 colic [13] and Simple colonic obstruction and distention (SCOD) colic [11] found

64 increased risk if the horse displayed CBWS behaviour. The temperament of the

65 horse, especially if irritable and excitable has been suggested to be a risk factor for

66 colic [14]. 'Stress' has also been implicated in equine gastric ulceration syndrome

67 which may also present with chronic intermittent colic [15]. However, the link

68 between behaviour and colic is likely not straightforward as horses who were easily

69 frightened, excited, went off food when stressed or had an inquisitive nature were

70 found to be at reduced risk in one study [16]. Objective measures of what constitutes

71 'stress' in the horse and how this translates into colic risk are subjects worthy of

72 further research.

73 This study aimed to identify risk factors for recurrent colic (including those factors

74 which may vary over time) among the veterinary-accessing general horse population.

75 Hypotheses were that diet and management, particularly stabling, grazing and

76 exercise; preventive health care (such as anthelmintic treatment and dentistry) and

77 behavioural aspects influence the risk of recurrent colic.

\section{Materials and Methods}

79 Study design and data collection

80 A nested case-control design was used with cases and unmatched controls randomly

81 selected from a previously described longitudinal cohort study [6]. Both veterinary

82 attended $(n=59)$ and owner reported $(n=17)$ recurrent colic episodes were included.

83 In brief, the cohort study enrolled 127 horses recruited via first opinion equine

84 veterinary surgeons in the North West of UK. These were horses that were diagnosed

85 with colic and had responded to medical treatment. Clinical details of the recruitment

86 colic and each subsequent colic episode were sought from the attending veterinary

87 surgeon. Horses with a history of surgery for colic, those less than 6 months old and

88 mares with foals at foot were excluded from the study. 
89 Owners completed a baseline and 3 follow up telephone questionnaires at

90 approximately 4 month intervals. The baseline questionnaire collected data on the

91 initial (recruitment) colic episode, the horses' signalment, use and behaviour,

92 prophylactic health care including vaccination, dentistry and deworming, and

93 management practices including diet, turnout and exercise routine.

94 The follow-up telephone questionnaires captured data that could potentially change

95 during the study period and recorded any further colic episodes. Information

96 regarding behaviour was not measured during follow-up, as it was considered that

97 this would change little over the course of the study. Participating owners were asked

98 to notify the study team if their horse had any further episodes of colic (including

99 any owner-reported colic episodes where a veterinary surgeon was not called). For

100 each recurrent colic episode, a further questionnaire was completed to ascertain: the

101 date of the colic, whether the recurrence satisfied the case definition, a description of

102 the colic, treatment and outcomes and whether the owner had any hypotheses of

103 cause and, what recommendations the attending veterinary surgeon had made.

104 Horses were censored if they died, had colic surgery or were lost to follow up. A

105 nested case-control study using data collected from this longitudinal cohort was

106 designed to analyse the effect of time-varying and non time-varying variables

107 occurring over the course of the study.

108 Case definition

109 Recurrent colic was defined as an episode of colic diagnosed by a veterinary surgeon

110 or, behavioural signs of colic observed (in the case of owner reported colic), and

111 which occurred more than 48 hours after a prior episode of colic on the proviso that

112 the horse had been free from signs of colic, eating a normal diet and passing normal

113 faeces for a full 48 hours. Behavioural signs suggestive of colic included one or

114 more of the following signs; flank watching, pawing, kicking belly, rolling, violent

115 rolling, repeated stretching as if to urinate, grinding teeth, flehemen/curling lip,

116 sweating, flatulence, getting up and down/restless and other.

\section{Selection of cases and controls}


Data were extracted from the cohort study and used to determine the effect of timevarying and non time-varying variables occurring over the course of the year by examining exposures in the 30 day period prior to being a case or control.

121 All recurrent colic episodes (total $n=59$ of which 17 were owner reported episodes) were selected as cases and the data relating to 30 days prior to the colic episode were used to determine exposure status. Three controls per case were randomly selected

124 from all horse-time at risk. All contributing time at risk was summated until the 125 point of loss to follow up, colic surgery or death due to colic or other reasons. Two

126 days from the recruitment episode, and 2 days subsequent to each episode of 127 recurrent colic were excluded from time at risk (according to our case definition).

128 Additionally, for each case of recurrent colic the 30 days prior to the colic could not 129 be selected as control data as these were contributory days to the 'case' time at risk 130 in line with other studies $[11,17]$.

131 From the remaining cumulative time at risk (44,301 days) [6] a list of computer 132 generated random numbers were used to select 177 control horse days. Case horses 133 could serve as controls before they became a case or when they were back 'at risk' 134 and a horse could be selected as a control at more than one time point. The selected 135 case and control days were transformed back into the corresponding dates for each 136 horse and exposure variables recorded within the 30 days prior to this date were 137 extracted.

139 Statistical analysis

140 Univariable analysis was conducted on all variables as listed in supplementary

141 information (see supplementary information tables $1 \& 2$ ). Categorical and 142 continuous variables were assessed using univaraible logistic regression (Minitab 143 15). The functional form of the relationship between continuous variables and the 144 outcome were tested for linearity using Generalised additive models (GAM) [18] and 145 plotted using S-Plus 2000. In order to assess the effect of changes in management 146 that occurred during the study (i.e. time-varying effects) data were extracted and 147 recategorised. This included a change in: the time spent at pasture; the frequency of 148 forage feeds; the frequency of concentrate feeds; time spent exercising and a change in stocking density between each of the follow up questionnaires. These variables 
were recoded and defined categorically as follows: no change occurred, an overall increase and an overall decrease between follow ups.

Prior to model building, all variables were assessed for correlation using Spearman's correlation coefficients. Any variables with $\mathrm{p}<0.2$ were included in a multivariable logistic regression model built manually using stepwise backwards elimination (SPSS). Variables were retained if their exclusion resulted in a likelihood ratio test statistic (LRTS) of $\mathrm{p}<0.05$ or if there was evidence of confounding. The resulting model was retested by forcing all variables (with $\mathrm{p}<0.2$ ) back into the model. The effect of biologically plausible interaction terms was tested in the model. Finally a multi-level multivariable model was built incorporating a random effect term for 'horse' (as a number of horses had multiple colic events during the cohort) to assess clustering within horse and the effect on the parameters of the model (MLwiN Version 2.18 Centre for Multilevel Modelling, University of Bristol). Model fit was assessed using the Hosmer-Lemeshow test statistic [19]. The stability of the model and the influence of individual data points were explored by assessment of delta-beta values for each variable in the final model. All other analysis except for multi-level modelling was conducted in SPSS and Minitab.

\section{Results}

In total 59 recurrent colic episodes (including 17 owner reported episodes) and 177 controls were included. The median time to first episode of recurrence was 101 days ( $\mathrm{n}=43, \min 2, \max 404$, IQ range 217 days). Three horses had a first recurrence within 7 days of the initial colic episode and seven horses had first recurrence within 30 days. There were 9 horses with 2 episodes of recurrence, 4 horses with 3 episodes, 2 horses with 4 episodes and 1 horse with 5 recurrent colic episodes during the course of the cohort. For further details of horse-time contributed to study see [6].

Tables $1 \& 2$ (within supplementary material) detail results of univariable analysis. A total of 47 variables were used to build the multivariable model. A multivariable logistic regression model (Table 1) showed that horses that displayed CBWS or weaving behaviours, had reduced time spent at pasture and were fed probiotics had 
an increased risk of colic. The variable "fed probiotics" was borderline significant $(\mathrm{p}=0.055)$ and the overall fit of the model with the data was improved judging by the Hosmer-Lemeshow test statistic hence it was retained within the model but should be interpreted with caution.

Time spent at pasture (in hours per day per week) was found to approximate a linear relationship with the risk of recurrence. Although the odds ratio was small (0.99) this represents the change in risk of colic occurring per hour/day change in grazing over the course of a week. For example, compared to a horse that is always stabled, a horse with 12 hours/day/week access to pasture had almost half the risk (OR=0.43) of recurrent colic (Table 2).

There was a significant interaction between feeding fruit/vegetables and CBWS behaviour. This suggested that among horses that displayed CBWS behaviour, those also fed fruit/vegetables were at reduced risk of recurrent colic $(\mathrm{OR}=0.15)$ compared to those that just demonstrated CBWS behaviour $(\mathrm{OR}=10.1)$ (see table 1 footnote).

A multilevel model, including horse as a random effect, showed there was no significant within-horse clustering and hence no effect on the other parameters within the model. Analysis of the delta beta distributions for the variables in the model found that the CBWS variable had one influential data point $(>0.4$ or -0.4$)$. This was a control horse and its removal resulted in a larger odds ratio for risk of CBWS $(16.3,95 \%$ CI $3.2,82.4)$ in the model. The data for this horse were correct and therefore were retained in the final analysis.

\section{Discussion}

This study has identified a number of factors associated with altered risk of recurrent colic in the general horse population. Currently, there is little information on the temporality of risk factors (i.e. changes that occur over time) and recurrent colic. One study called for a 'clearer definition of time relationships of events with colic' in order that specific management recommendations could be made [8].

The use of a nested case-control design within a cohort study enabled particular time-varying and non time-varying exposures to be examined. In contrast to many previously published case-control studies on colic, the exposures were recorded 
212 prospectively (i.e. prior to a horse having a recurrent colic episode). This creates

213 some logistical disadvantages as data collection is time consuming and involves

214 regular contact with participants throughout the study period, and losses to follow up

215 may occur more frequently due to the extended nature of the study (although our

216 participant retention rate was good 89.8\%). However, these potential disadvantages

217 are outweighed by the benefits of obtaining likely better quality of data as recall bias

218 should be minimised. Using this type of study design, it was also possible to

219 examine a specific time period prior to the recurrent colic episode. The findings of

220 the present study builds upon those reported from the baseline survey [6] as it allows

221 for the variability that occurs within the management of the horse over the course of

222 a year and accounts for each individual episode of colic.

223 Horses that display CBWS behaviour have previously been shown to be at increased 224 risk of medical colic and some types of surgical colic lesions $[6,11,17 \& 20]$. The 225 aetiology of this stereotypic behaviour is not fully understood and many hypotheses

226 have been proposed including confinement within a stable [21], reduced access to

227 pasture along with high concentrate and low forage diets [22], horse temperament,

228 other stressors, genetics or a form of gastrointestinal dysfunction [17 \& 23]. CBWS

229 may not only be a marker for particular individuals that are prone to colic, it may

230 have a direct physical influence upon colic aetiology or could be a proxy for other

231 management factors involved in colic risk. Ultimately the link between CBWS

232 behaviour and colic remains unclear and warrants further investigation.

233

234 This study is the first to report an association between weaving behaviour and

235 increased risk of recurrent colic. Eleven (8.7\%) horses among the cohort displayed

236 this form of behaviour, 7 of which had a colic recurrence (63.6\% of horses that

237 weaved). Whereas CBWS behaviours are oral stereotypies, weaving is classed as a

238 locomotor stereotypy and is likely to have different predisposing causes. Hypotheses

239 of why horses may develop this behaviour include confinement within a stable,

240 reduced opportunity for social contact or an anticipatory/frustration element [24 \&

$24125]$. One study found that the type and quantity of forage provided, bedding, yard

242 size and tactile contact with neighbouring horses while stabled influenced the

243 occurrence of weaving [21]. It may be that horses that weaved in our cohort had

244 differing aspects of their general management compared to those that did not weave 
or that aspects of their management may be linked to both weaving and colic risk, although this an area requiring further investigation.

Increasing time spent at pasture was found to be associated with a decreased risk of recurrent colic in the present study. This is in agreement with three previous studies investigating the risk of colic $[11,17 \& 26]$ and may reflect the beneficial effects of grazing perhaps in combination with the ability to exercise and opportunities to interact with the natural environment. The equine gastrointestinal tract has evolved to be optimally adapted for trickle feeding and a forage based diet. Providing suitable access to pasture may go some way to meet these physiological requirements however additional attention may be required to ensure pasture is appropriately managed in order to balance out the management of colic risk and other medical conditions. This may include; pasture analysis to examine whether suitable grass types and other plant species are present, limiting parasitic exposure, and maintaining an appropriate stocking density as a few examples. Within this study, 'access to pasture' included only those horses that were turned out onto grass paddocks/fields and we found no association with acreage or stocking density and the risk of recurrence. Any change in the amount of time spent at pasture is likely to constitute a change in diet, itself a risk factor for colic. For this reason it is advisable that any changes are introduced slowly.

Feeding fruit and vegetables was found to be associated with a decreased risk of colic within this cohort although this finding should be interpreted with caution and may not be applicable to other populations. The exposure was recorded as a binary variable (feed fruit/veg yes/no) and it was not possible to assess the effect of frequency, type or quantity fed due to small numbers within the sample. Therefore it is not possible to make recommendations on how to incorporate fruit and vegetables into the diet to alter colic risk. One possibility is this may be a proxy measure for dietary practices of particular types of owners or other management factors not accounted for within these data. This finding requires additional research in order to understand the impact of feeding fruit and vegetables on colic risk in practice. One study investigating feeding preferences in horses found that some fruit and vegetable flavours increased the acceptance and rate of consumption of food [27]. If such feeding behaviour is interpreted by owners as the horse enjoying the food then this 
may motivate some owners to provide such foods within the diet. Further work is warranted to examine dietary decisions made by different types of horse-owners and to understand the motivations behind feeding behaviours and their potential impact on equine health.

282 A significant interaction was found whereby the increased risk associated with 283 CBWS was modified by additionally feeding fruit and vegetables. It may be for 284 example that feeding fruit and vegetables is associated with particular management 285 factors such as catching horses in the field, and the availability of turn out could 286 potentially link this finding with CBWS although it is unclear from this data how to 287 interpret this effect. This finding adds credence to the possible protective role of 288 feeding fruit/vegetables reported above; however it is not possible to recommend how to incorporate these into the diet from this data. A Chi-square test indicated that 290 those horses fed fruit/vegetables were no less likely to display CBWS behaviour than 291 those not fed fruit/vegetables. Another study found a significant interaction between 292 crib-biting and carer of the horse and proposed that this may be due to the way in 293 which particular types of carers approach the management of horses [16]. It may be that carers differ in their motivations and decision making for the types of food offered to their horses and this may be influenced by their attitudes surrounding the needs of horses with CBWS behaviour.

297 The finding that probiotics may be associated with an increased risk of recurrent colic should be interpreted with caution. The confidence intervals cross one and this variable was not statistically significant $(\mathrm{p}=0.055)$ but its inclusion improved the overall fit of the model. It is possible that administering probiotics is a marker for certain types of horses that already have an inherent risk of colic e.g. older horses, those with dental problems, those prone to laminitis or with previous episodes of 303 colic. There is little evidence on the effect of feeding probiotics on equine colic. 304 Although not directly comparable to this study of an adult equine population (where a variety of different probiotics were used), one study reported the administration of 306 Lactobacillus pentosus WE7 preparation to be associated with diarrhoea and signs of colic in foals [28]. Another study reported a beneficial effect with feeding probiotics to horses with acute enterocolitis leading to a reduction in hospitalisation time [29].

309 Nevertheless, questions remain about the efficacy of probiotic preparations and 310 further clinical trials are required to investigate their therapeutic potential in horses 
especially as many preparations are available via the veterinary market [30] and appear popular among some owners.

One of the study limitations was that it was not possible to identify the cause of colic in most cases. This is commonly the case among medical colic treated in general practice with one study reporting $72 \%$ of colic cases seen over 2 years were spasmodic/undiagnosed colic [3]. The underlying cause of such undiagnosed colic may be multifactorial with a range of presentations [31]. It is possible that misclassification bias may have been introduced where owner reported colic cases were included in the analysis. However, all further episodes of colic (or suspected colic for the owner reported episodes) were assessed according to whether the described episode fitted the case criteria for colic (as outlined within the methods). It was considered that the combination of the fulfilment of the case criteria, assessment of the reported signs of colic (see [6] for further details) along with the owners' experience of a veterinary diagnosed medical colic would improve the assumption that these owner reported colic episodes were correctly reported in order to reduce this potential bias. Within this study we did not find an association with exercise (type or frequency) and the risk of recurrence. Additionally, we did not collect blood or faecal samples from this cohort for parasitological investigations. Previous studies have suggested a link between gastrointestinal parasites (e.g. tapeworm associated colic [32] or more rarely, thromboembolic disease / verminous arteritis caused by Strongylus vulgaris [31] associated with recurrent colic). We measured factors that may be considered proxy measures for parasitic exposure such as use of anthelmintic products (frequencies and types); pasture management approaches, stocking density, co-grazing and some exploration of how owners decide upon approaches to parasite control. However, none of these factors were influential in the final model.

\section{Conclusions and practical relevance}

Horses that have experienced a colic episode are known to be at increased risk of future episodes. In this study we report risk factors that provide further evidence to support managemental approaches to colic prevention namely the provision of access to grazing. The behavioural risk factors identified (CBWS and weaving) highlight individual horses that may be at higher risk for recurrent colic and therefore it would seem appropriate that particular attention is paid to their colic prevention strategies 
343 (e.g. diet, access to grazing, anthelmintic prophylaxis and dental care). Further

344 studies to investigate the mechanisms by which these behaviours influence the risk

345 of colic are warranted.

346 Table 1: Multivariable logistic regression model of risk factors associated with recurrence of colic in the general horse population in North West England

\begin{tabular}{|l|l|l|l|l|l|}
\hline & Category & $\begin{array}{l}\text { Odds } \\
\text { ratio }\end{array}$ & $\begin{array}{l}\text { Lower } \\
\mathbf{9 5 \%} \\
\text { confidence } \\
\text { interval }\end{array}$ & $\begin{array}{l}\text { Upper } \\
\text { 95\% } \\
\text { confidence } \\
\text { interval }\end{array}$ & $\begin{array}{l}\text { Likelihood } \\
\text { ratio p- } \\
\text { value }\end{array}$ \\
\hline $\begin{array}{l}\text { Crib-biting or } \\
\text { windsucking } \\
\text { (CBWS) } \\
\text { behaviour }\end{array}$ & No (Ref) & 1.0 & & & \\
\hline & Yes & 10.1 & 2.5 & 41.0 & $<0.001$ \\
\hline $\begin{array}{l}\text { Weaving } \\
\text { behaviour }\end{array}$ & No (Ref) & 1.0 & & & \\
\hline & Yes & 3.9 & 1.5 & 10.1 & 0.004 \\
\hline $\begin{array}{l}\text { Time spent at } \\
\text { pasture (hours per } \\
\text { day / week) }\end{array}$ & 0.99 & 0.99 & 1 & 0.005 \\
\hline $\begin{array}{l}\text { Fruit / vegetables } \\
\text { in diet }\end{array}$ & No (Ref) & 1.0 & & & \\
\hline & Yes & 0.5 & 0.2 & 1.3 & 0.16 \\
\hline Probiotics in diet & No (Ref) & 1.0 & & & \\
\hline & Yes & 2.4 & 0.99 & 6.0 & 0.06 \\
\hline $\begin{array}{l}\text { Interaction term: } \\
\text { Windsucking x } \\
\text { fruit/vegetables in } \\
\text { diet }\end{array}$ & & 0.03 & 0.002 & 0.5 & 0.005 \\
\hline Based on 56 cases & & & & & \\
\hline
\end{tabular}

Based on 56 cases and 159 controls. Hosmer-Lemeshow test statistic $\mathrm{p}=0.446$.

349 Ref=Reference category

350 Explanation of interaction term

- No fruit/vegetables and horse does not display CBWS behaviour, OR = 1

- No fruit/vegetables and horse displays CBWS behaviour, OR $=10.1$

- Yes fruit/vegetables and horse does not display CBWS behaviour, OR = 0.5

- Yes fruit/vegetables and horse displays CBWS behaviour, $\mathrm{OR}=(10.1 \times 0.5$ $\mathrm{x} 0.03)=0.15$

Table 2: Decreasing risk of recurrent colic with time spent at pasture

\begin{tabular}{|l|c|c|}
\hline Time spent at pasture & $\begin{array}{c}\text { Number of hours } \\
\text { per week }\end{array}$ & $\begin{array}{c}\text { Odds ratio of risk of } \\
\text { recurrent colic }\end{array}$ \\
\hline
\end{tabular}




\begin{tabular}{|l|c|c|}
\hline 1 hour / day / week & 7 & 0.93 \\
\hline 3 hrs / day / week & 21 & 0.81 \\
\hline 6 hrs / day / week & 42 & 0.66 \\
\hline 12 hours / day / week & 84 & 0.43 \\
\hline 24 hours / day / week & 168 & 0.18 \\
\hline
\end{tabular}

\section{References}

[1] Mellor, D.J., Love, S., Walker, R., Gettinby, G. and Reid, S.W.J. (2001) Sentinel practice-based survey of the management and health of horses in Northern Britain. Vet. Rec. 149, 417-423.

[2] Mair, T. S. (2004) BEVA evidence-based medicine colic survey. In: Proceedings British Equine Veterinary Association Congress Birmingham, UK 58-59.

[3] Proudman, C. J. (1991). A 2-year prospective survey of equine colic in general practice.

Equine vet. J. 24(2), 90-93.

[4] Hart, S. and Southwood, L.L. (2010) The enigma of post operative recurrent colic:

372 Challenges with diagnosis and management. Equine vet. J. 22 (8), 408-411.

374 [5] Beard, W.L. and Freeman, D.E. (1994) Clinical case conference. J. Am. vet. med. Ass. $375 \quad 204,1165$.

[6] Detail to be provided upon acceptance.

378 [7] Archer, D. C. and Proudman, C.J. (2006) Epidemiological clues to preventing colic. The 379 Vet. J. 172(1), 29-39.

380 [8] Tinker, M.K, White, N.A., Lessard, P., Thatcher, C.D., Pelzer, K.D., Davis, B. and 381 Carmel, K. (1997) Prospective study of equine colic risk factors. Equine vet. J. 29 (6), 454382458.

384 [9] Cohen, N.D., Gibbs, P.G. and Woods, A.M. (1999) Dietary and other management 385 factors associated with colic in horses. J. Am. vet. med. Ass. 215 (1), 53-60. 
388 study of the association between various management factors and development of colic in

389 horses. J. Am. vet. med. Ass. 206(5), 667-673.

[11] Hillyer, M. H., Taylor, F. G. R., Proudman, C. J., Edwards, G. B., Smith, J. E., and

[12] Cohen, N. D. and Peloso, J.G. (1996) Risk factors for history of previous colic and for

[13] Archer, D. C., Proudman, C.J., Pinchbeck, G., Smith, J.E., French, N.P. and Edwards,

G.B. (2004) Entrapment of the small intestine in the epiploic foramen in horses: a retrospective analysis of 71 cases recorded between 1991 and 2001. Vet. Rec. 155(25), 7937.

402

[14] Reeves, M. J., Salman, M. D. and Smith, G. (1996) Risk factors for equine acute abdominal disease (colic): Results from a multi-center case-control study. Prev. vet. Med. 26(3-4), 285-301. [15] Nadeau, J.A. and Andrews, F.M. (2009) Equine gastric ulcer syndrome: The continuing conundrum. Equine vet. J. 41 (7), 611-615.

409

410 [16] Archer D.C., Pinchbeck, G.L., French, N.P. and Proudman, C.J. (2008) Risk factors for epiploic foramen entrapment colic: an international study. Equine vet. J. 40 (3), 224-230.

413 [17] Archer, D.C., Pinchbeck, G.L., French, N.P. and Proudman, C.J. (2008) Risk factors for 414 epiploic foramen entrapment colic in a UK horse population: A prospective case-control 415 study. Equine. Vet. J. 40 (4), 405-410.

417 [18] Hatsie, T. and Tibshirani, R. (1986) Generalized Additive Models. Statistical Sciences 1 418 (3), 297-310.

419 [19] Dahoo, I., Martin, W. and Stryhn, H. (2003) Veterinary Epidemiologic Research AVC 420 Inc. University of Prince Edward Island p 360. 
422 [20] Malamed, R., Beger, J., Bain, M.J., Kass, P. and Spier, S.J. (2010) Retrospective

423 evaluation of crib-biting and windsucking behaviours and owner-perceived behavioural

424 traits as risk factors for colic in horses. Equine vet. J. 42 (8), 686-692.

425

[21] McGreevy, P.D., Cripps, P.J., French, N.P., Green, L.E. and Nicol, C.J. (1995)

427 Management factors associated with stereotypic and redirected behaviour in the

428 Thoroughbred horse. Equine vet. J. 27 (2), 86-91.

429

430

[22] Redbo, I., Redbo - Torstensson, P., Oldberg, F.O., Hedendahl, A. and Holme, J. (1998)

Factors affecting behavioural disturbances in race-horses. Anim. Sci. 66, 475-481.

[23] Escalona, E., Merrifield, C., Proudman, C., Holmes, E. (2012) Metabolomics of

Thoroughbred racehorses: a pilot study. Abstract at $9^{\text {th }}$ Annual International

Conference of the Metabolomics Society.

436

437

438

[24] Nicol, C. (1999) Understanding equine stereotypies. Equine vet. J. 28, 20-25.

[25] Mills, D.S. and Nankervis, K.J. (1999) Equine behaviour: Principles and practice

[26] Hudson, J.M., Cohen, N.D., Gibbs, P.G. and Thompson, J.A. (2001) Feeding practices

[27] Goodwin, D., Davidson, H.P.B. and Harris, P. (2005) Selection and acceptance of

[28] Weese, J.S. and Rousseau, J. (2005) Evaluation of Lactobacillus pentosus WE7 for of Saccharomyces boulardii for treatment of horses with acute enterocolitis. J. Am. Vet. 
458 [31] Hillyer, M. H. and Mair, T. S. (1997) Recurrent colic in the mature horse: a

459 retrospective review of 58 cases. Equine vet. J. 29 (6), 421-4.

460

461 [32] Proudman, C.J. \& Holdstock, N.B. (2000) Investigation of an outbreak of tapeworm-

462 associated colic in a training yard. Equine Vet. J. supp 32, 37-41.

463

464 Supplementary material

465 Table 1: Univariable results of risk factors associated with recurrence of colic $(\mathrm{p}<0.2)$.

466 Table 2: Univariable results of factors found to have no significant association with

467 recurrence of colic ( $p$-value $>0.2$ )

468 